\title{
Sémaphorines \\ et cancers : \\ état des lieux
}

Patrick Nasarre, Bruno Constantin,

$>$ Les sémaphorines jouent un rôle central dans Harry A. Drabkin, Joëlle Roche

le guidage axonal, la régénération axonale chez l'adulte et le développement de certains tissus. De plus, la découverte de leur implication dans l'angiogenèse et la réponse immunitaire indique une fonction beaucoup plus large de ces protéines. En 1996, trois études concomitantes ont permis de cloner les gènes codant pour deux de ces protéines, SEMA3B et SEMA3F, dans la région 3p21.3 qui subit de fréquentes pertes d'hétérozygotie dans les cancers du sein et du poumon. L'hypothèse avait alors été émise selon laquelle ces gènes pouvaient être des suppresseurs de tumeurs. Les études ultérieures ont confirmé ce rôle. À l'inverse, d'autres sémaphorines stimuleraient la croissance tumorale. Cet article fait le point sur les dernières connaissances acquises concernant le rôle des sémaphorines dans les cancers. II souligne en particulier les propriétés anti-migratoires et antiangiogéniques de ces molécules, qui pourraient jouer un rôle déterminant dans l'inhibition de la formation de métastases. <

Dans cet article, seront présentés dans une première partie la famille des sémaphorines, leurs récepteurs, leur signalisation, puis leurs principales fonctions dans les tissus sains. La partie suivante traitera du rôle de certaines sémaphorines dans les cancers en tant que molécules pro- ou anti-tumorales.

\section{Les sémaphorines : récepteurs, signalisation et fonctions}

Le terme «sémaphorine » vient du grec semâ, «signal », et phoros, «qui porte», en raison du rôle de ces molécules dans le guidage axonal découvert il y a douze ans. La famille des sémaphorines compte environ 25 membres répartis en huit classes, dont une classe virale (Figure 1) [1]. Les sémaphorines peuvent

Article reçu le 5 janvier 2005, accepté le 21 février 2005.

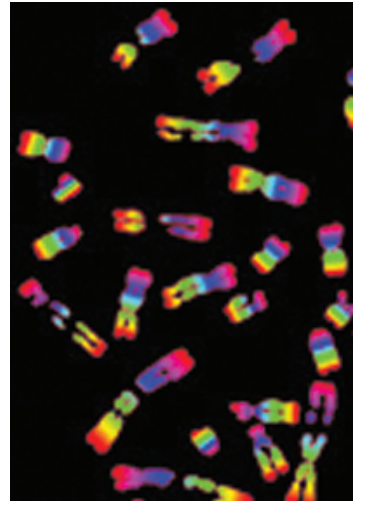

être soit attachées à la membrane par un groupement glycosyl phosphatidyl inositol

(GPI), soit transmembranaires ou sécrétées, mais elles possèdent toutes en commun un domaine caractéristique de 500 acides aminés riche en résidus cystéine appelé domaine «Sema». Ce domaine est également retrouvé dans la séquence des récepteurs des facteurs de dispersion, les récepteurs Met et Ron qui participent à la régulation de l'invasion cellulaire [2]. Les premiers récepteurs des sémaphorines identifiés, les neuropilines 1 et 2 (NRPl et NRP2), se lient spécifiquement aux sémaphorines de classe $3[3,4]$. La partie intracytoplasmique des neuropilines est cependant extrêmement courte, et d'autres partenaires protéiques capables d'activer la signalisation des sémaphorines ont été recherchés. Les plexines ont ainsi été identifiées et longtemps proposées comme uniques molécules capables d'activer la signalisation intracellulaire des sémaphorines [2, 5]. Le modèle faisant intervenir les neuropilines dans le complexe récepteur des sémaphorines de classe 3 vient cependant d'être remis en question puisque $S E M A 3 \varepsilon$, chez la souris, exerce un effet répulsif sur les cellules endothéliales en se liant directement à la plexine Dl [6]. De même, SEMA3B ne se lierait pas aux cellules exprimant NRP1 ou NRP2 seulement [6], ce qui suggère l'intervention d'autres récepteurs. Les récepteurs de la sémaphorine SEMA5A seraient également, plutôt qu'une plexine, des glycosaminoglycanes 
de type héparane et chondroïtine sulfates qui seraient susceptibles de convertir l'effet répulsif de SEMA5A en effet attractif sur les axones [7]. Très récemment, de nouvelles molécules impliquées dans l'adhérence cellulaire, telles que la protéine Ll-CAM (pour revue, voir [8]) et les intégrines [9, 10], ont été associées au complexe récepteur des sémaphorines (Figure 2). Une fonction prépondérante des sémaphorines serait de réguler l'expression ou l'activité de protéines intervenant dans la formation de contacts intercellulaires ou de contacts entre cellules et matrice extracellulaire. La voie de signalisation de certaines sémaphorines a fait l'objet d'études approfondies, mais reste encore largement inconnue (pour revue, voir [11]) (Figure 2). Les premières molécules découvertes dans la voie de signalisation des sémaphorines sont les CRMP (collapsin response mediator proteins). Cependant, leur position exacte dans la signalisation des sémaphorines n'est pas encore établie. Les petites protéines $G$ de la famille Rho participeraient, quant à elles, à l'activation des plexines en réponse à la liaison de sémaphorines de classe 3 à leur complexe récepteur. Des études sur une lignée cellulaire de médulloblastome [12] et de carcinome mammaire [13] suggèrent que les protéines MAPK ERK1 et ERK2, MAPK p38, GSK3 et PI3K sont, pour leur part, impliquées dans la signalisation de SEMA3A.

D'un point de vue fonctionnel, les sémaphorines participent au positionnement des neurones, à la formation des faisceaux d'axones et au guidage des axones et des dendrites au cours du développement (pour revue, voir [14]). Chez l'adulte, les sémaphorines seraient également impliquées dans l'inhibition de la régénération axonale après lésion [15, 16]. Par ailleurs, elles seraient impliquées dans le développement du poumon, du cœur, des tissus osseux et rénal et il a été observé qu'elles jouent également un rôle central dans la réponse immunitaire chez l'adulte. Cependant, la découverte de leur rôle possible dans l'angiogenèse est probablement celle qui a motivé l'étude du rôle des sémaphorines dans les cancers. Plusieurs études d'invalidation des gènes codant pour les sémaphorines ou les neuropilines ont permis de révéler de graves altérations vasculaires et cardiaques conduisant souvent à la mort des mutants au cours du développement embryonnaire $[17,18]$. Les neuropilines interagissent avec le facteur de croissance des cellules endothéliales, le VEGF ${ }_{165}$ (vascular endothelial growth factor). Cette liaison a pour conséquence d'augmenter l'affinité du VEGF ${ }_{165}$ pour son propre récepteur, le VEGFR-2, stimulant ainsi l'angiogenèse [19]. Des études complémentaires de compétition entre VEGF et sémaphorines en culture ont révélé que certaines sémaphorines de classe 3 pourraient jouer un rôle anti-migratoire et antiangiogénique majeur [20]. Par ces propriétés, les sémaphorines de classe 3 limiteraient la croissance tumorale et empêcheraient la diffusion de métastases.

\section{Fonction des sémaphorines dans les cancers}

Les premières études visant à tester l'implication des sémaphorines dans les cancers sont relativement récentes et ont connu un essor important ces cinq dernières années. Même si l'implication réelle des sémaphorines n'a pas été démontrée dans tous les types tumoraux étudiés, certaines d'entre elles auraient un rôle protumoral alors que d'autres, au contraire, auraient un rôle anti-tumoral (Tableau I). Seule la sémaphorine SEMA4D/CD100 pourrait avoir un effet pro- ou antitumoral variant selon le type de tissu tumoral étudié.

\section{Sémaphorines pro-tumorales}

L'une des premières sémaphorines identifiées comme molécule pouvant avoir un rôle pro-tumoral est SEMA3C. Son expression augmente dans des lignées de carcinomes ovariens résistantes au CDDP (cis-diaminedichloroplatine) et l'induction d'une surexpression de SEMA3C dans les cellules de carcinomes ovariens augmente leur résistance au CDDP [21]. Une autre sémaphorine de classe 3, SEMA3E, est fortement exprimée dans des lignées cellulaires d'adénocarcinomes mammaires métastasiques, alors qu'elle n'est exprimée que dans $30 \%$ des lignées cellulaires non métastasiques [22]. Une sémaphorine de classe 6 a également été impliquée dans la formation des tumeurs. Le traitement de deux lignées cellulaires de glioblastomes par l'acide rétinoïque tout-trans provo-

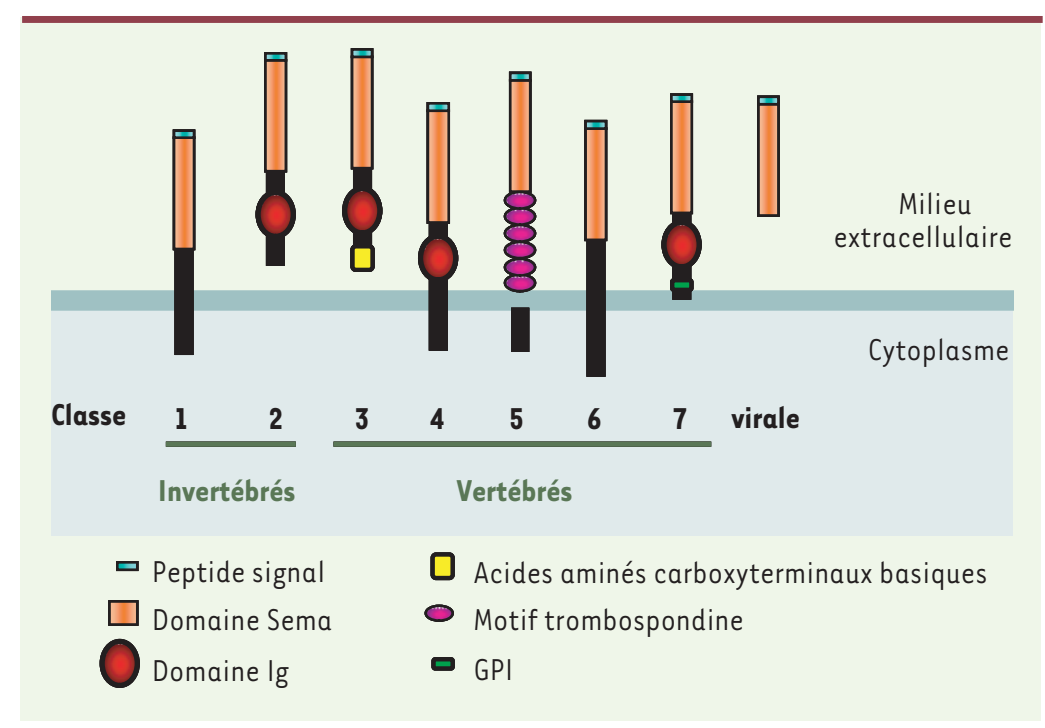

Figure 1. Présentation schématique des huit classes de la famille des sémaphorines. Les sémaphorines de classe 3, qui jouent un rôle important dans les cancers, possèdent, outre le domaine Sema, un domaine de type immunoglobuline (Ig) et une partie carboxyterminale riche en acides aminés basiques. GPI : groupement glycosyl phosphatidyl inositol (selon [1]). 
que, en effet, une diminution de l'expression de SEMA6B de sept à dix fois, ce qui suggère que cette sémaphorine serait un facteur promoteur de tumeurs [23]. Une autre sémaphorine transmembranaire, SEMA4D/CD100, serait fortement exprimée dans les leucémies lymphocytaires chroniques [24]. Par son rôle promoteur de prolifération et inducteur de survie, SEMA4D/CD100 serait, dans ce type de leucémies, un promoteur de tumeurs. De plus, cette sémaphorine contrôle l'invasion cellulaire en se couplant à Met [25] et stimule l'angiogenèse [26, 27]. Enfin, certains travaux suggèrent que la sémaphorine transmembranaire de classe 5 , SEMA5C, serait impliquée dans la croissance tumorale dans un modèle tumoral chez la drosophile [28]. Les études précédemment décrites suggèrent qu'il existe une corrélation entre le niveau d'expression de certaines sémaphorines et le degré d'agressivité des lignées tumorales en culture. En revanche, aucun modèle animal n'a été établi, ce qui n'a pas permis de démontrer leur rôle pro-tumoral.

\section{Sémaphorines anti-tumorales}

Si le rôle des sémaphorines protumorales n'est pas encore clairement défini, celui des sémaphorines anti-tumorales, en revanche, a fait l'objet d'études récentes importantes qui ont permis de mieux comprendre leur mode d'action. Un rôle suppresseur de tumeur de deux sémaphorines de classe 3 (SEMA3B et SEMA3F) a été proposé (pour revue, voir $[29,30]$ ) comme cela a été décrit plus haut, à la suite du clonage de leurs gènes. Cette hypothèse fut considérablement renforcée par les expériences du groupe de $S$. Naylor qui a montré qu'un clone $\mathrm{Pl}$ de $80 \mathrm{~kb}$ contenant le gène SEMA3F supprime la formation de tumeurs induites par des cellules A9 de fibrosarcomes murins injectées chez la souris athymique [31]. Plus récemment, l'expression de SEMA3F a été étudiée dans plus de 100 échantillons de tumeurs pulmonaires humaines et dans 50 lésions prénéoplasiques [32, 33]. Ces études ont montré que la perte d'expression de SEMA3F est un phénomène précoce dans la cancérogenèse. De plus, cette perte d'expression est inversement corrélée au degré d'agressivité des tumeurs, et quand SEMA3F reste exprimée dans les cellules transformées, elle est délocalisée dans le cytoplasme alors qu'elle est enrichie dans la membrane des cellules épithéliales pulmonaires d'un tissu sain. À l'inverse, le VEGF est surexprimé au cours du développement des tumeurs et détecté à la membrane des cellules. Ces observations nous ont ainsi amenés à proposer une compétition entre VEGF et SEMA3F pour la fixation à leurs récepteurs communs, les neuropilines. De nombreuses observations en culture sont également en faveur d'un rôle antitumoral

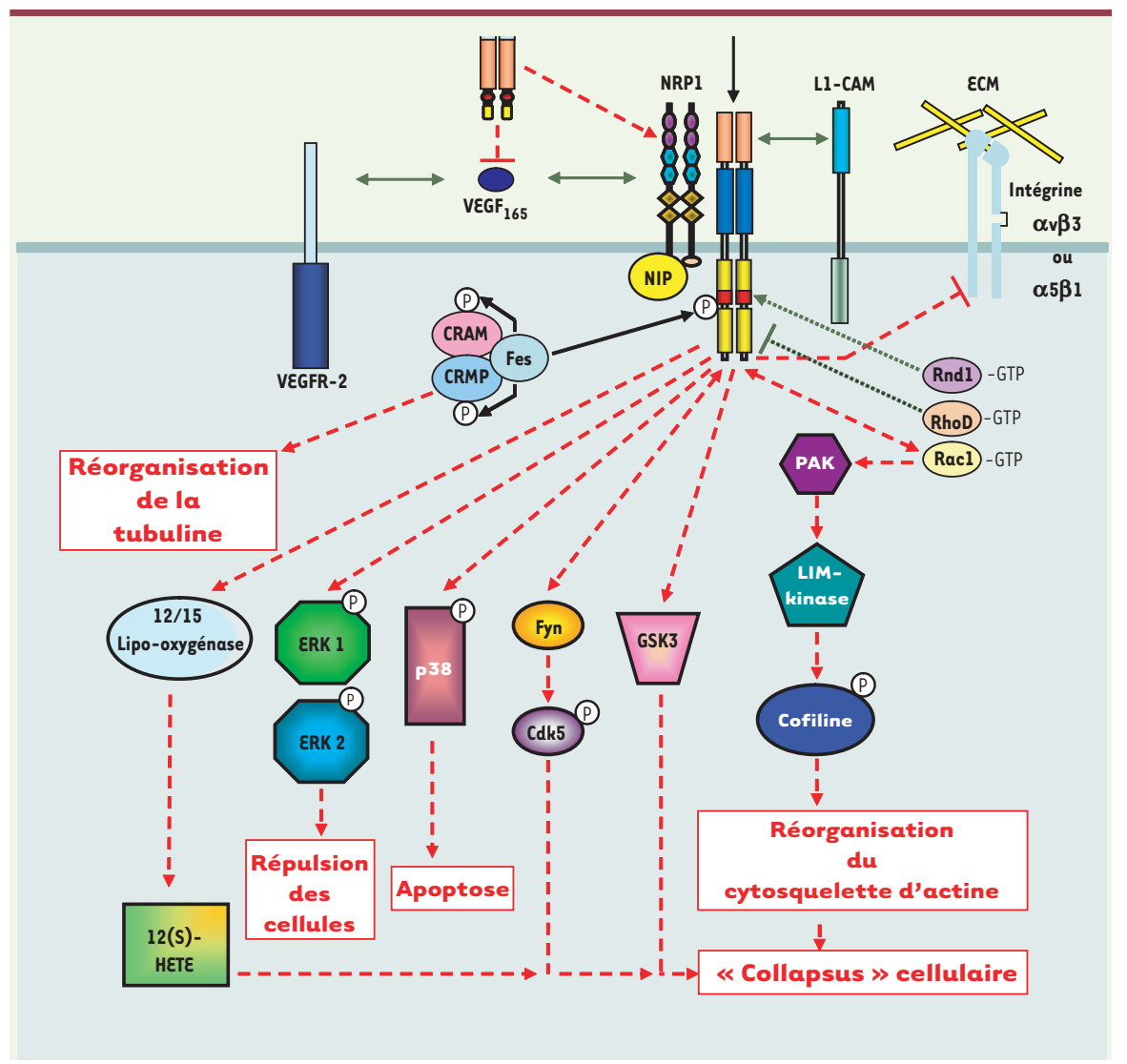

Figure 2. Principales molécules impliquées dans la voie de signalisation de SEMA3A et effets induits par l'activation de ces molécules. SEMA3A entre en compétition avec le VEGF ${ }_{165}$ pour la liaison aux neuropilines. La liaison de SEMA3A dimérisée à son complexe récepteur (incluant les neuropilines [NRP], les plexines [Plex] et éventuellement Ll-CAM et les intégrines associées à la matrice extracellulaire $[\varepsilon C M])$ favorise le recrutement ou l'activation de plusieurs molécules: NIP (NRPI-interacting protein); CRAM (CRMP-associated molecule); CRMP (collapsin response mediator protein); Fes; 12/15 lipo-oxygénase ; 12(S)-HETE (12 [S]- et 15 [S]-hydroperoxyeicosatetraenoic acid) ; عRK $1 / 2$ (extracellular regulated kinase); p38 MAPK (p38 mitogen activated protein kinase) ; Fyn ; Cdk5 (cyclin-dependant kinase); GSK3 (glycogen synthase kinase); PAK (p21-activated kinase); LIMkinase; Cofiline. Les petites protéines $G$ de la famille Rho interviennent à la fois dans l'activation des plexines et dans la transmission du signal à la cellule (traits pointillés verts). Les différentes voies représentées (flèches pointillées rouges) seraient impliquées dans la réorganisation des cytosquelettes d'actine et de tubuline, régulant ainsi l'étalement et la migration cellulaire, et dans l'apoptose induite par SEMA3A. 
de SEMA3F. SEMA3F réduit d'environ $30 \%$ la capacité de cellules $\mathrm{NCl}-\mathrm{H} 1299$ de carcinomes pulmonaires à former des colonies [34], et l'application de SEMA3F exogène à des lignées de carcinomes mammaires réduit leur prolifération [35]. Ces mêmes lignées, après un traitement par SEMA3F, montrent également une diminution de l'étalement ainsi qu'une réduction de l'émission de prolongements membranaires suggérant une régulation de la motilité cellulaire $[35,36]$. Un gradient de SEMA3F a un effet répulsif sur les cellules migrantes qui expriment NRP2 [35]. Par ailleurs, SEMA3F provoque une diminution du nombre de contacts intercellulaires sans effet pro-migratoire. Cet effet implique une diminution de l'expression de la cadhérine $\varepsilon$ et une délocalisation dans le cytoplasme des cellules de la cadhérine $\varepsilon$ et de la $\beta$-caténine [35]. Les études in situ montrent que la surexpression de SEMA3F dans les cellules HEY de carcinomes ovariens [37], dans les cellules A9 de fibrosarcomes murins [37] ou dans les cellules HEK 293 [38] ralentit la croissance tumorale après injection chez l'animal. De plus, nous avons observé que la surexpression de SEMA3F dans les cellules H157 de carcinome pulmonaire ralentit la croissance tumorale après injection orthotopique dans le poumon de rats immunodéficients [39]. Dans les tumeurs provoquées par des cellules HEK 293 transfectées par SEMA3F, la densité vasculaire est fortement réduite [38]. Dernièrement, le groupe de M. Klagsbrun a testé les effets d'une surexpression de SEMA3F dans la lignée cellulaire SM de mélanome [40]. Lorsque ces cellules surexprimant SEMA3F sont injectées sous la peau de souris immunodéficientes, elle forment des tumeurs encapsulées dont la périphérie est entourée de fibroblastes et de collagène. De plus, aucune métastase n'est retrouvée dans le poumon et les ganglions lymphatiques des souris. À l'inverse, après injection des cellules SM témoins, les tumeurs formées ne sont pas encapsulées et une hyperplasie importante des kératinocytes qui entourent les tumeurs est observée. L'étude approfondie des

\begin{tabular}{|c|c|c|}
\hline & Pro-tumorales & Anti-tumorales \\
\hline Profil d'expression & $\begin{array}{l}\text { SEMA4D : exprimée dans cellules B } \\
\text { (leucémies lymphocytaires chroniques) } \\
\text { [24] } \\
\text { SEMA3C : expression augmentée dans les } \\
\text { carcinomes ovariens résistants au CDDP } \\
\text { [21] } \\
\text { SEMA3E : expression forte dans les lignées } \\
\text { d'adénocarcinomes mammaires } \\
\text { métastasiques [22] } \\
\text { SEMA6B : expression diminuée dans une } \\
\text { lignée de glioblastome traitée par l'acide } \\
\text { rétinoïque tout-trans [23] }\end{array}$ & $\begin{array}{l}\text { SEMA4D : pas d'expression dans les cellules B } \\
\text { (lymphomes non hodgkiniens) [47] } \\
\text { SEMA3B : expression réduite dans les tumeurs } \\
\text { pulmonaires [55] } \\
\text { SEMA3F : niveau d'expression inversement } \\
\text { corrélé au degré d'agressivité des tumeurs } \\
\text { dans le poumon [32, 33], variable dans les } \\
\text { lignées pulmonaires [55] }\end{array}$ \\
\hline Modèle de culture cellulaire & $\begin{array}{l}\text { SEMA3C : stimulation de la survie cellulaire } \\
\text { [21] }\end{array}$ & $\begin{array}{l}\text { SEMA3A : induction de l'apoptose [12], inhibition } \\
\text { de la migration cellulaire }[13,20,46] \text {, répulsion } \\
\text { cellulaire [12] } \\
\text { SEMA3B : induction de l'apoptose [34, 41-43], } \\
\text { inhibition de la prolifération cellulaire [34] } \\
\text { SEMA3F : inhibition de la prolifération cellulaire } \\
\text { et répulsion cellulaire }[35,36]\end{array}$ \\
\hline Modèle animal & $\begin{array}{l}\text { SEMA5C : promoteur de croissance chez la } \\
\text { drosophile [28] }\end{array}$ & $\begin{array}{l}\text { SEMA3B : inhibition de la croissance tumorale } \\
\text { [43] } \\
\text { SEMA3F : inhibition de la croissance tumorale } \\
\text { [37-39], inhibition de la néo-vascularisation des } \\
\text { tumeurs [38-40], inhibition des métastases [40] }\end{array}$ \\
\hline
\end{tabular}

Tableau I. Rôle présumé de certaines sémaphorines dans la tumorigenèse. Seul l'effet anti-tumoral des sémaphorines de classe 3 a été validé à ce jour par des modèles animaux. L'effet pro-tumoral de certaines sémaphorines, quoique suggéré, reste à être démontré chez l'animal. CDPP : cis-diaminedichloroplatine. 
tumeurs induites pas les cellules de mélanome transfectées par SEMA3F révèle une importante diminution du diamètre, du degré de ramification et de la quantité de vaisseaux au sein de ces tumeurs. Finalement, les études du groupe de G. Neufeld [38] et de celui de M. Klagsbrun [40] suggèrent que l'effet anti-tumoral de SEMA3F serait essentiellement dû à son activité anti-angiogénique.

Comme SEMA3F, et même de façon plus efficace, SEMA3B réduit la capacité des lignées tumorales pulmonaires à former des colonies en culture [34]. Cependant, alors que cela n'a pas été montré pour SEMA3F, SEMA3B induit une augmentation de l'apoptose. De plus, l'expression de SEMA3B est stimulée d'un facteur 8 par la protéine p 53 et le site de liaison de $p 53$ dans la séquence du gène $S E M A 3 B$

\section{A}
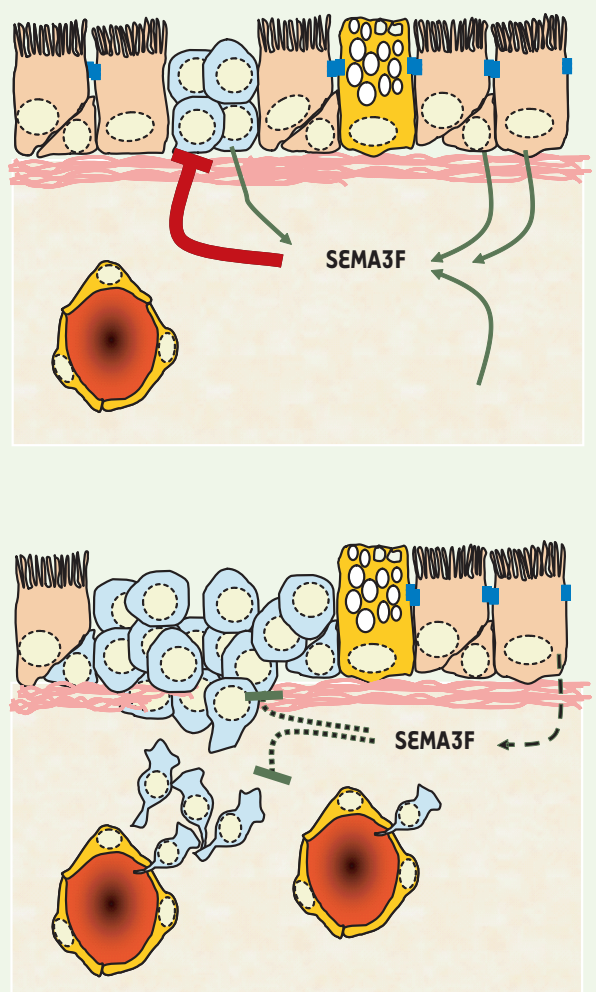

Figure 3. Rôle anti-tumoral de SEMA3F dans le poumon. A. L'expression de SEMA3F dans le tissu épithélial pulmonaire (cellules épithéliales en orange, cellule sécrétrice de mucus en jaune) et dans les lésions cancéreuses précoces empêcherait la migration des cellules tumorales (en bleu). B. En revanche, au cours du développement des tumeurs, la perte de SEMA3F lèverait cette inhibition. Les cellules tumorales pourraient alors franchir la membrane basale, envahir les tissus et pénétrer dans les vaisseaux sanguins (en rouge). À ce stade, l'angiogenèse est stimulée dans la tumeur. $a$ été identifié [41]. Récemment, il a été observé que le $\mathrm{VEGF}_{165}$ inhibe l'effet pro-apoptotique et anti-mitotique induit par SEMA3B sur des lignées de carcinomes pulmonaires et mammaires [42]. La surexpression de SEMA3B dans des cellules HEY de carcinomes ovariens injectées à des souris immunodéficientes ralentit de manière drastique la progression tumorale [43]. Ainsi, SEMA3B, comme SEMA3F, aurait un rôle anti-tumoral, mais, à la différence de SEMA3F, SEMA3B inhiberait la croissance tumorale en stimulant l'apoptose.

Les gènes suppresseurs de tumeurs se caractérisent généralement par l'existence de nombreuses mutations. La recherche de mutations de $S E M A 3 B$ et $S E M A 3 F$ n'a permis de détecter que de rares mutations pour $S E M A 3 B$ et aucune mutation pour SEMA3F dans des lignées tumorales pulmonaires $[29,44]$. L'expression de SEMA3B serait réprimée par méthylation de I'ADN dans son promoteur $[34,45]$ et la reconfiguration de la chromatine semble être un élément important dans la régulation de SEMA3F.

Quant à SEMA3A, elle pourrait également avoir un rôle antitumoral, bien que ses effets n'aient été étudiés qu'en culture. En effet, SEMA3A induit une répulsion des cellules de la lignée DEV de médulloblastome et, après une exposition prolongée à cette sémaphorine, les cellules meurent par apoptose [12]. L'inhibition de l'expression de NRPl ou de SEMA3A par la technique d'interférence par l'ARN augmente par ailleurs les capacités migratoires de cellules de carcinomes mammaires [46]. En revanche, SEMA3A provoque le «collapsus » des cellules de l'une de ces lignées de carcinomes mammaires [13]. In vivo, SEMA3A pourrait donc exercer un effet anti-migratoire et pro-apoptotique sur les cellules tumorales.

Enfin, la sémaphorine SEMA4D/CD100 ne serait plus exprimée à la surface des cellules lymphomateuses de lymphomes non hodgkiniens à cellules B [47]. Comme SEMA4D/CD100 favorise l'agrégation et l'adhérence des lymphocytes, il est proposé que, dans les cellules $B$, cette fonction serait inhibée avec la perte d'expression de SEMA4D/ CD100, limitant ainsi la réponse anti-tumorale des cellules T. Dans les lymphomes non hodgkiniens, et contrairement aux leucémies lymphocytaires chroniques, SEMA4D/CD100 pourrait alors avoir un rôle anti-tumoral.

\section{Neuropilines}

Outre les sémaphorines, les neuropilines ont été impliquées dans les cancers en tant que récepteurs du VEGF. Ainsi, leur expression augmente dans les ostéosarcomes, les neuroblastomes, les cancers du poumon et les adénocarcinomes pancréatiques et du côlon. De plus, la surexpression de NRPI dans une lignée de carcinome de la prostate provoque une augmentation de la taille des tumeurs et de leur vascularisation après injection des cellules à des rats immunodéficients [48]. Ces tumeurs fixent également une plus grande quantité de VEGF. A l'inverse, lorsque les cellules surexpriment une forme soluble de NRP1, sNRP1, capable de se lier au $V G_{165}$, les tumeurs formées après injection sont hémorragiques, moins vascularisées et présentent un taux d'apoptose plus élevé [49]. Les neuropilines pourraient donc être utilisées comme marqueur dans de nombreux cancers et l'augmentation de leur expression serait associée à un pronostic vital réservé. 


\section{CRMP}

Une étude récente indique que le niveau d'expression de CRMPI (collapsin response mediator protein 1) serait inversement corrélé au degré d'agressivité de tumeurs pulmonaires et que l'inhibition de son expression favoriserait l'invasion cellulaire [50]. De plus, l'expression de CRMPI serait induite par le CTGF (connective tissue growth factor), dont le niveau d'expression diminue dans les tumeurs pulmonaires. Les CRMP, activées par les sémaphorines, joueraient donc un rôle important dans l'inhibition de la croissance tumorale.

\section{Conclusions}

Par leurs propriétés anti-migratoires et anti-angiogéniques (Figure 3), certaines sémaphorines pourraient se révéler être de bons candidats pour la recherche de nouvelles molécules anti-tumorales. Cependant, la plupart des résultats obtenus jusqu'à présent concernent surtout les sémaphorines de classe 3 pour lesquelles des modèles animaux ont été réalisés. Pour les autres sémaphorines, des corrélations entre le niveau d'expression et le degré d'agressivité des cellules tumorales ont été observées, mais aucune démonstration de leur rôle n'a été faite chez l'animal. Il est important de noter que, comme dans le système nerveux, les sémaphorines jouent un double rôle dans les cancers, promoteur ou inhibiteur de la croissance tumorale. II faut également souligner qu'outre les sémaphorines, d'autres molécules intervenant dans le guidage axonal telles que la protéine SLIT2 [51] et son récepteur « Roundabout » ou « Robo », les éphrines [52] ou les nétrines [53] et leurs récepteurs [54] ont récemment été impliquées dans la tumorigenèse. La régulation fine de leur expression dans l'espace et dans le temps ne servirait donc pas uniquement à l'établissement et au maintien des connexions neuronales. Les études futures devront déterminer le rôle de chacune de ces molécules dans les tissus concernés. $\diamond$

\section{REMERCIEMENTS}

Les auteurs remercient La Ligue Nationale contre le Cancer (Comités de la Vienne, de la Charente et de la Charente Maritime), l'Association pour la Recherche contre le Cancer et la Société Française du Cancer pour leur soutien sur le projet «SEMA3F et cancer».

\section{SUMMARY}

Semaphorins and cancers : an up'dating

Semaphorins, first described as axon guidance molecules, play an essential role in neural development, angiogenesis and immunological response. In 1996, two semaphorin genes, SEMA3B and SEMA3F, were isolated from chromosomal region 3 p21.3 believed to contain a tumor suppressor gene based on frequent loss of heterozygosity in lung and breast cancer. Since these first studies, several semaphorins have been involved in tumor progression. Some semaphorins have been proposed to have pro-tumoral properties, whereas others have been shown to have tumor suppressive activity. This review summarizes the most recent data implicating semaphorins in cancers. $\diamond$

\section{RÉFÉRENCES}

1. Unified nomenclature for the semaphorins/collapsins. Nomenclature committee. Cell $1999 ; 97: 551-2$.

2. Winberg ML, Noordemeer JN, Tamagnone L, et al. Plexin A is a neuronal semaphorin receptor that controls axon guidance. Cell 1998; 95 : 903-16.

3. Kolodkin AL, Levengood DV, Rowe EG, et al. Neuropilin is a semaphorin III receptor Cell $1997 ; 90: 753-62$.

4. Chen $\mathrm{H}$, Chedotal A, He Z, et al. Neuropilin-2, a novel member of the neuropilin family, is a high affinity receptor for the semaphorins Sema $\varepsilon$ and Sema IV but not Sema III. Neuron 1997 ; 19 : 547-59.

5. Tamagnone L, Artigiani S, Chen H, et al. Plexins are a large family of receptors for transmembrane, secreted, and GPI-anchored semaphorins in vertebrates. Cell $1999 ; 99: 356-61$

6. Gu C, Yoshida Y, Livet J, et al. Semaphorin $3 \varepsilon$ and Plexin-DI control vascular pattern independently of neuropilins. Science $2005 ; 307: 265-8$.

7. Kanter DB, Chivatakarn 0 , Peer KL, et al. Semaphorin $5 \mathrm{~A}$ is a bifunctional axon guidance cue regulated by heparan and chondroitin sulfate proteoglycans. Neuron $2004 ; 44: 961-75$.

8. Castellani V, Rougon G. Control of semaphorin signaling. Curr Opin Neurobiol 2002 ; $12: 532-41$

9. Serini G, Valdembri D, Zanivan S, et al. Class 3 semaphorins control vascular morphogenesis by inhibiting integrin function. Nature 2003; 424 : 391-7.

10. Pasterkamp R, Peschon J, Spriggs M, Kolodkin A. Semaphorin 7A promotes axon outgrowth through integrins and MAPKs. Nature $2003 ; 424: 398-405$.

11. Pasterkamp R, Kolodkin A. Semaphorin junction : macking tracks toward neural connectivity. Curr Opin Neurobiol $2003 ; 13: 79-89$.

12. Bagnard D, Sainturet N, Meyronet D, et al. Differential MAP kinases activation during Semaphorin3A-induced repulsion or apoptosis of neural progenitor cells. Mol Cell Neurosci $2004 ; 25: 722-31$.

13. Eickholt BJ, Walsh FS, Doherty P. An inactive pool of GSK-3 at the leading edge of growth cones is implicated in Semaphorin 3A signaling. J Cell Biol 2002; $157: 211$ 7.

14. He Z, Wang KC, Koprivica V, et al. Knowing how to navigate : mechanisms of signaling in the nervous system. Sci STKE $2002 ; 119$ : RE1.

15. Skutella T, Nitsch R. New molecules for hippocampal development. Trends Neurosci $2001 ; 24: 107-13$.

16. Gavazzi I. Semaphorin-neuropilin-1 interactions in plasticity and regeneration of adult neurons. Cell Tissue Res $2001 ; 305: 275-84$.

17. Behar 0 , Golden JA, Mashimo H, et al. Semaphorin III is needed for normal patterning and growth of nerves, bones and heart. Nature $1996 ; 383: 525-8$.

18. Takashima S, Kitakaze M, Asakura M, et al. Targeting of both mouse neuropilin-1 and neuropilin-2 genes severely impairs development yolk sac and embryonic angiogenesis. Proc Natl Acad Sci USA 2002 ; 99 : 3657-62.

19. Soker S, Takashima S, Miao H, et al. Neuropilin-1 is expressed by endothelial and tumor cells as an isoform-specific receptor for vascular endothelial growth factor. Cell $1998 ; 92: 735-45$.

20. Miao HQ, Soker S, Feiner L, et al. Neuropilin-1 mediates collapsin-1/semaphorin III inhibition of endothelial cell motility: Functional competition of collapsin-1 and vascular endothelial growth factor-165. J Cell Biol 1999; $146: 233-42$.

21. Konno R. Gene expression profiling of human ovarian epithelial tumors by digo nucleotide microarray. Hum Cell 2001 ; 14 : 261-6.

22. Christensen CR, Klingelhofer J, Tarabykina S, et al. Transcription of a novel mouse semaphorin gene, $\mathrm{M}$-semaH, correlates with the metastatic ability of mouse tumor cell lines. Cancer Res $1998 ; 58: 1238-44$.

23. Correa RG, Sasahara RM, Bengton MH, et al. Human semaphorin 6B ([HSA]SEMA6B), a novel human class 6 semaphorin gene: Alternative splicing and all-trans-retinoic aciddependent downregulation in glioblastoma cell lines. Genomics $2001 ; 73$ : 343-8.

24. Granziero L, Circosta P, Scielzo C, et al. CD100/Plexin-Bl interactions sustain proliferation and survival of normal and leukemic CD5 $B$ lymphocytes. Blood 2003; $101: 1962-9$.

25. Giordano $S$, Corso $S$, Conrotto $P$, et al. The semaphorin $4 \mathrm{D}$ receptor controls invasive growth by coupling with Met. Nat Cell Biol $2002 ; 4: 720-4$.

26. Basile J, Barac A, Zhu T, et al. Class IV semaphorins promote angiogenesis by stimulating Rho-initiated pathways through plexin-B. Cancer Res 2004 ; 65 : 5212-24. 
27. Conrotto P, Valdembri D, Corso $S$, et al. Sema4D induces angiogenesis through Met recruitment by plexin Bl. Blood 2005, 4 janvier, online.

28. Woodhouse $\varepsilon$, Fisher $A$, Bandle R, et al. Drosophila screening model for metastasis : Semaphorin $5 c$ is required for l(2) gl cancer phenotype. Proc Natl Acad Sci USA 2003; $100: 11463-8$.

29. Lerman MI, Minna JD. The 630-kb lung cancer homozygous deletion region on human chromosome 3p21.3 : identification and evaluation of the resident candidate tumor suppressor genes. The international lung cancer chromosome 3 p21.3 tumor suppressor gene consortium. Cancer Res 2000; 60:6116-33.

30. Roche J, West J, Gemmill R, Drabkin H. Chromosome 3p, gènes suppresseurs de tumeurs et gènes de sémaphorines en 3p21.3. Med Sci (Paris) 1998; 14 : 283-90.

31. Todd M, Xiang R, Garcia D, et al. An $80 \mathrm{~Kb} \mathrm{Pl} \mathrm{clone} \mathrm{from} \mathrm{chromosome} \mathrm{3p21.3}$ suppresses tumor growth in vivo. Oncogene 1996; 13:2387-96.

32. Brambilla $\varepsilon$, Constantin B, Drabkin H, Roche J. Semaphorin SEMA3F localization in malignant human lung and cell lines: A suggested role in cell adhesion and cell migration. Am J Pathol $2000 ; 156: 939-50$.

33. Lantuéjoul S, Constantin B, Drabkin H, et al. Expression of VEGF, semaphorin SEMA3F, and their common receptors neuropilins NP1 and NP2 in preinvasive bronchial lesions, lung tumors, and cell lines. J Pathol 2003 ; 200 : 336-47.

34. Tomizawa $Y$, Sekido $Y$, Kondo M, et al. Inhibition of lung cancer cell growth and induction of apoptosis after reexpression of $3 p 21.3$ candidate tumor suppressor gene SEMA3B. Proc Natl Acad Sci USA 2001 ; 98 : 13954-9.

35. Nasarre $P$, Kusy $S$, Constantin B, et al. Semaphorin SEMA3F has a repulsing activity on breast cancer cells and inhibits $\varepsilon$-cadherin-mediated cell adhesion. Neoplasia 2005 (sous presse).

36. Nasarre P, Constantin B, Rouhaud L, et al. Semaphorin SEMA3F and VEGF have opposing effects on cell attachment and spreading. Neoplasia $2003 ; 5: 83-92$.

37. Xiang R, Davalos AR, Hensel CH, et al. Semaphorin 3F gene from human 3p21.3 suppresses tumor formation in nude mice. Cancer Res $2002 ; 62: 2637-43$.

38. Kessler 0 , Shraga-Heled N, Lange T, et al. Semaphorin-3F is an inhibitor of tumor angiogenesis. Cancer Res 2004 ; 64 : 1008-15.

39. Kusy S, Nasarre P, Chan D, et al. Selective suppression of in vivo tumorigenicity by semaphorin SEMA3F in lung cancer cells. Neoplasia 2005 (sous presse).

40. Bielenberg DR, Hida Y, Shimizu A, et al. Semaphorin 3F, a chemorepulsant for endothelial cells, induces a poorly vascularized, encapsulated, nonmetastatic tumor phenotype. J Clin Invest 2004 ; 114 : 1260-71.

41. Ochi K, Mori T, Toyama Y, et al. Identification of semaphorin3B as a direct target of p53. Neoplasia $2002 ; 4: 82-7$.

42. Castro-Rivera $\varepsilon$, Ran S, Thorpe P, Minna JD. Semaphorin 3B (SEMA3B) induces apoptosis in lung and breast cancer, whereas VEGF165 antagonizes this effect. Proc Natl Acad Sci USA $2004 ; 101$ : 11432-7.
43. Tse C, Xiang RH, Bracht T, Naylor SL. Human Semaphorin 3B (SEMA3B) located at chromosome 3 p21.3 suppresses tumor formation in an adenocarcinoma cell line. Cancer Res $2002 ; 62$ : 542-6.

44. Xiang R, Hensel C, Garcia D, et al. Isolation of the human semaphorin III/F gene (SEMA3F) at chromosome 3p21, a region deleted in lung cancer. Genomics 1996; 32 : 39-48.

45. Kuroki T, Trapasso F, Yendamuri S, et al. Allelic loss on chromosome 3p21.3 and promoter hypermethylation of semaphorin $3 \mathrm{~B}$ in non-small cell lung cancer. Cancer Res $2003 ; 63$ : 3352-5.

46. Bachelder RE, Lipscomb $\varepsilon A$, Lin X, et al. Competing autocrine pathways involving alternative neuropilin-1 ligands regulate chemotaxis of carcinoma cells. Cancer Res $2003 ; 63: 5230-3$

47. Dorfman D, Shahsafaei A, Nadler L, Freeman G. The leucocyte semaphorin CD100 is expressed in most T-cell, but few B-cell, non-Hodgkin's lymphomas. Am J Pathol 1998 ; $153: 255-62$.

48. Miao $\mathrm{HQ}$, Lee $\mathrm{P}$, Lin $\mathrm{H}$, et al. Neuropilin-l expression by tumor cells promotes tumor angiogenesis and progression. FASEB J $2000 ; 14: 2532-9$.

49. Gagnon ML, Bielenberg DR, Gechtman Z, et al. Identification of a natural soluble neuropilin-1 that binds vascular endothelial growth factor : In vivo expression and antitumor activity. Proc Natl Acad Sci USA $2000 ; 97: 2573-8$.

50. Chang CC, Shih JY, Jeng YM, et al. Connective tissue growth factor and its role in lung adenocarcinoma invasion and metastasis. J Natl Cancer Inst 2004 ; 96 : 364-75.

51. Prasad A, Fernandis AZ, Rao Y, Ganju K. Slit protein-mediated inhibition of CXCR4induced chemotactic and chemoinvasive signaling pathways in breast cancer cells. J Biol Chem $2004 ; 279$ : 9115-24.

52. Surawska H, Ma PC, Salgia R. The role of ephrins and $\varepsilon p h$ receptors in cancer. Cytokine Growth Factor Rev 2004 ; 15 : 419-33.

53. Arakawa H. Netrin-1 and its receptors in tumorigenesis. Nat Rev Cancer 2004 ; 4 : 978 87.

54. Lu X, Le Noble F, Yuan L, et al. The netrin receptor UNC5B mediates guidance events controlling morphogenesis of the vascular system. Nature $2004 ; 432: 179-86$.

55. Sekido Y, Bader S, Latif F, et al. Human semaphorins A(V) and IV reside in the $3 p 21.3$ small cell lung cancer deletion region and demonstrate distinct expression patterns. Proc Natl Acad Sci USA $1996 ; 93: 4120-5$.

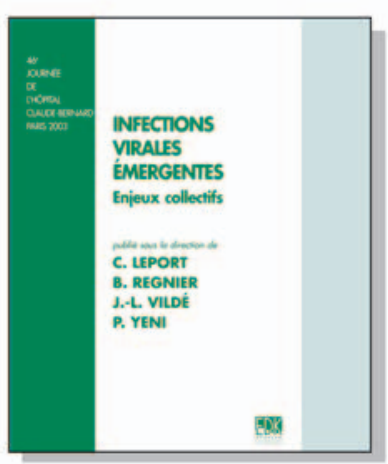

ISBN : 2-84254-092-1 148 pages

Un ouvrage remarquable qui fait le point sur les infections virales émergentes, la préven. tion et les traitements: infections SIV et risque de zoonoses, SRAS, autres viroses res. piratoires et grippe, Biotex (variole), arsovroses, fièvres hémorragiques virales, Ebola
a
Infections virales émergentes Enjeux collectifs

\section{Bon de commande}

À retourner à EDK, 10 Villa d'Orléans - 75014 PARIS

Tél. : 0153910606 - Fax : 0153910607 - E-mail : edk@edk.fr

NOM : Prénom :

Adresse :

Code postal :

Ville :

Pays :

Fonction :

Je souhaite recevoir Y'ouvrage Infections virales émergentes : $20 €+3 €$ de port $=23 €$ TTC

en __exemplaire, soit un total de _... $€$

$\square$ Par chèque, à l'ordre de E D K

$\square$ Par carte bancaire:

$\square$ Visa $\square$ Eurocard/Mastercard

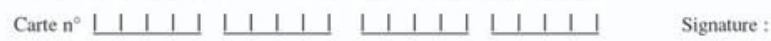

TIRÉS À PART

J. Roche 